\title{
Microvessel Density and Expression of Vascular Endothelial Growth Factor and Its Receptors in Diffuse Large B-Cell Lymphoma Subtypes
}

\author{
Dita Gratzinger, ${ }^{*}$ Shuchun Zhao, ${ }^{*}$ \\ Robert J. Marinelli, ${ }^{\dagger}$ Amy V. Kapp, ${ }^{\ddagger}$ \\ Robert J. Tibshirani, ${ }^{\ddagger}$ Anne S. Hammer, ${ }^{\S}$ \\ Stephen Hamilton-Dutoit, ${ }^{\S}$ and \\ Yasodha Natkunam* \\ From the Departments of Pathology, ${ }^{*}$ Biochemistry, ${ }^{\dagger}$ \\ and Health Research and Policy, ${ }^{\ddagger}$ Stanford University, Stanford, \\ California; and Institute of Pathology, ${ }^{\circledR}$ Aarbus University \\ Hospital, Aarbus, Denmark
}

Angiogenesis is known to play a major role in neoplasia, including hematolymphoid neoplasia. We assessed the relationships among angiogenesis and expression of vascular endothelial growth factor and its receptors in the context of clinically and biologically relevant subtypes of diffuse large B-cell lymphoma using immunohistochemical evaluation of tissue microarrays. We found that diffuse large B-cell lymphoma specimens showing higher local vascular endothelial growth factor expression showed correspondingly higher microvessel density, implying that lymphoma cells induce local tumor angiogenesis. In addition, local vascular endothelial growth factor expression was higher in those specimens showing higher expression of the receptors of the growth factor, suggesting an autocrine growth-promoting feedback loop. The germinal center-like and nongerminal center-like subtypes of diffuse large Bcell lymphoma were biologically and prognostically distinct. Interestingly, only in the more clinically aggressive nongerminal center-like subtype were microvessel densities significantly higher in specimens showing higher vascular endothelial growth factor expression; the same was true for the finding of higher vascular endothelial growth factor receptor-1 expression in conjunction with higher vascular endothelial growth factor expression. These differences may have important implications for the responsiveness of the two diffuse large B-cell lymphoma subtypes to anti-vascular endothelial growth factor and anti-an-

\author{
giogenic therapies. (AmJ Pathol 2007, 170:1362-1369; DOI: \\ 10.2353/ajpath.2007.060901)
}

Angiogenesis and the proangiogenic growth factor vascular endothelial growth factor (VEGF; also known as vascular permeability factor) have a known role in solid neoplasia, and there is increasing evidence that they also play a role in hematolymphoid neoplasia. Increased microvessel density has been noted in a range of hematolymphoid disorders, including multiple myeloma, nonHodgkin lymphoma, acute and chronic leukemias of myeloid and lymphoid lineages, and myelodysplastic disorders. ${ }^{1}$ VEGF promotes angiogenesis and vascular permeability via its receptor VEGF receptor (VEGFR)-2 (also known as Flk-1). ${ }^{2}$ In addition, VEGF also has a direct role in hematolymphoid cell development; an autocrine loop involving VEGF and its receptor VEGFR-1 (also known as Flt-1) modulates in vivo hematopoietic stem cell survival and proliferation. ${ }^{3}$ Cell lines derived from a variety of hematolymphoid malignancies have been shown to express both VEGF and VEGFR-1, ${ }^{4}$ suggesting a role for a similar autocrine loop in neoplasia.

We assessed the interaction among microvessel density and local expression of VEGF and VEGFR-1 and -2 in diffuse large B-cell lymphoma (DLBCL). We further examined these findings in the context of clinically and biologically relevant subtypes of DLBCL. DLBCL is both common and aggressive, with a high mortality within the 1st year after diagnosis and 5-year overall survival of less than $50 \%{ }^{5}$ Recently, gene expression profiling has uncovered at least two biologically and prognostically distinct subgroups of DLBCL. ${ }^{6}$ These two subgroups are defined by the expression of sets of genes that they have in common with germinal center B cells (GC-like subtype) and activated peripheral blood $B$ cells (ABC or non-GClike subtype).

Supported in part by National Institutes of Health grants CA109335 and CA34233.

Accepted for publication January 4, 2007.

Address reprint requests to Dita Gratzinger, 300 Pasteur Dr., Lane 235, Stanford, CA 94305-5324. E-mail: ditag@stanford.edu. 
Although gene expression profiling is crucial in the research setting for identifying biologically relevant gene products, immunohistochemical analysis has the advantages of being readily available in the clinical setting and of enabling morphological evaluation of expression patterns and such higher order features as tumor vascularity. We have used immunohistochemistry to assess tissue microarrays of DLBCL for microvessel density (highlighted with the vascular marker CD34) and expression of VEGF and its receptors VEGFR-1 and VEGFR-2. We report the relationships among these variables both within the group of DLBCL cases as a whole and within the GC-like and non-GC-like subgroups individually.

\section{Materials and Methods}

\section{Tissue Microarray Construction}

A tissue microarray containing 94 DLBCL cases from the Institute of Pathology at Aarhus University Hospital (Aarhus, Denmark) was used for this study. Only de novo DLBCL cases were included. The cases were not collected consecutively. No cases of primary mediastinal DLBCL, intravascular DLBCL, T-cell or histiocyte-rich Bcell lymphoma, or other World Health Organization-recognized special variants of DLBCL were included. Tissue microarrays were previously constructed as described previously ${ }^{7}$ using a tissue arrayer (Beecher Instruments, Silver Spring, MD). ${ }^{8}$ Institutional review board approval was obtained for these studies.

\section{Immunohistochemistry}

Immunohistochemistry for CD34 (1:10 dilution; Becton Dickinson Biosciences, San Jose, CA), VEGFR-1 (1:50;
Lab Vision Corp., Fremont, CA), VEGFR-2 (1:400 dilution; Santa Cruz Biotechnology, Santa Cruz, CA), and VEGF (1:200 dilution; Santa Cruz Biotechnology) was performed on $4-\mu \mathrm{m}$ sections, which were placed on glass slides, baked for 1 hour at $60^{\circ} \mathrm{C}$, deparaffinized in xylene, and hydrated in a graded series of alcohol. Antigen retrieval was as follows: mild heat retrieval for CD34, ethylenediamine tetraacetic acid retrieval for VEGF, Tris retrieval for VEGFR-1, and Tris retrieval for VEGFR-2. Endogenous peroxidase was blocked, and the chromogen used was diaminobenzidine. Immunohistochemistry for HGAL, BCL2, BCL6, CD10, and MUM1 (also known as IRF4) was previously described. ${ }^{7}$ Images of the immunostained tetramethylammonium slides for CD34, VEGF, VEGFR-1, and VEGFR-2 were scanned, digitized, and stored at http://tma.stanford.edu/tma_portal/mvd (Stanford University School of Medicine, accessible as of 8/1/2006)

\section{Scoring of Immunohistochemical Stains}

Immunohistochemical stains for HGAL, BCL2, BCL6, CD10, MUM1,VEGFR-1, VEGFR-2, and VEGF were scored as follows based on the percentage of lymphoma cells stained: more than $30 \%$ staining was scored strong positive; 5 to $30 \%$ staining was scored weak positive; and less than $5 \%$ staining was scored negative. Staining was performed on two separate cores from each case of DLBCL; for each of the stains listed, at least 86 were evaluated on two duplicate cores, and at least 88 were evaluated on a single core. Where two scores were available for cores taken from the same specimen, the higher value was used in the analysis. For microvessel density quantitation, where two scores were available for cores taken from the same specimen, the average was used in

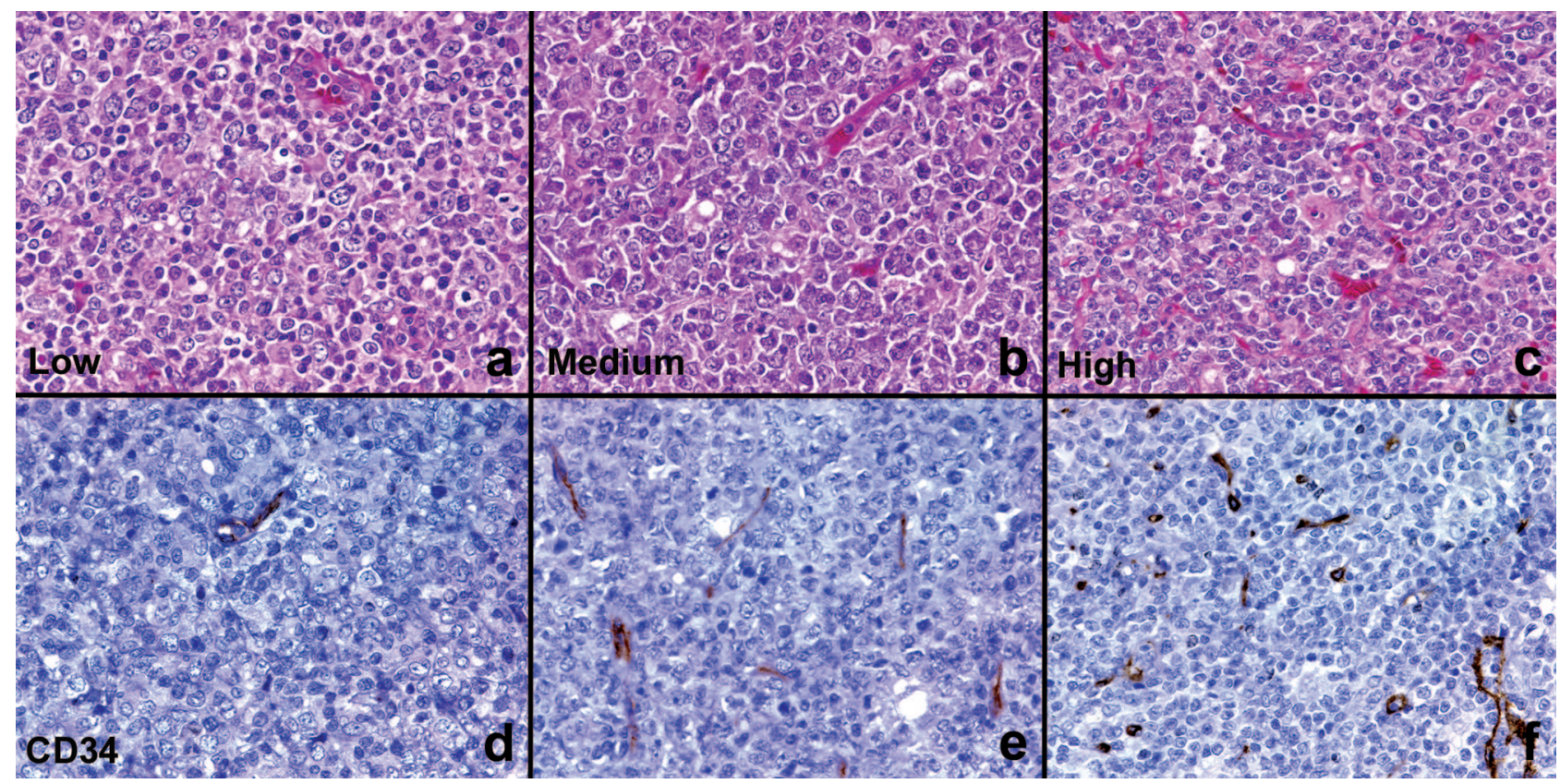

Figure 1. Vascularity in DLBCL. Examples of DLBCL illustrating low (a and $\mathbf{d}$ ), medium (b and e), and high (c and $\mathbf{f}$ ) vascularity (H\&E, anti-CD34; magnification, $\times 400)$. 
the analysis. The core was not evaluated if diagnostic tissue was not present or evaluation was precluded by technical problems such as high background staining. Scoring of immunostains was performed independently by D.G. (a hematopathology fellow) and checked by Y.N. (a hematopathologist), with resolution of the rare disagreements by doublescoping.

\section{Microvessel Density Counting}

Microvessel densities were evaluated by counting the number of $\mathrm{CD} 34^{+}$microvessels in the entire $1.0-\mathrm{mm}$ core at $\times 300$ ( $\times 20$ lens, $\times 15$ ocular) using an Olympus BX45 microscope (Center Valley, PA). A microvessel was defined as any distinct $\mathrm{CD} 34^{+}$cell or cell cluster, with no requirement for a vessel lumen. ${ }^{9}$ Cores were not evaluated if a portion of the core was missing or diagnostic tissue was not present in the entire core. Of 94 DLBCL cases, 87 had at least one core countable, and 80 had both cores countable. Scoring was performed by D.G. with independent spot-checking by Y.N. A subset of randomized lymphoma cores were blindly rescored from scanned images, and duplicate pairs were found to have similar vessel counts (data not shown).

\section{Hierarchical Cluster Analysis}

A coding scheme was used to encode immunohistochemical staining scores, with 0 for negative, 1 for uninterpretable/missing, 2 for weak, and 3 for strong staining. For clustering and visualization, these scores were mapped into the range ( -2 to 2$)$, with -2 for negative (green), 1 for weak, and 2 for strong positive (red). Microvessel density was scored by counting and encoded by setting the average count to 0 and scaling the counts above and below average into the range ( -2 to 2 ), visualized as green for low counts and red for high counts. Data were evaluated with nonsupervised hierarchical clustering and were visualized as heatmaps with Treeview. ${ }^{10}$ Specifically, Eisen's Cluster software ${ }^{11}$ was used to perform nonsupervised hierarchial clustering using uncentered Pearson correlation and average linkage. For the clustering used to identify GC-like and non-GC-like DLBCL specimens, the dendrogram was cut at the first split to create the two groups, as indicated in Figure 5.

\section{Results}

\section{Vascularity and Expression of VEGF by DLBCL}

The vascularity of the lymphomas was variable: the average microvessel count from any single DLBCL core ranged from 0 to 144 (Figure 1, a-f). A histogram highlights the wide distribution of microvessel densities in DLBCL (Figure 2a), with a relatively even distribution of lymphomas over the lower half of the vascularity range and fewer very highly vascular DLBCL. To assess how representative the microvessel counts within 1.0-mm cores are of the overall microvessel density of each lymphoma, the microvessel counts for each of the pairs of cores were plotted against
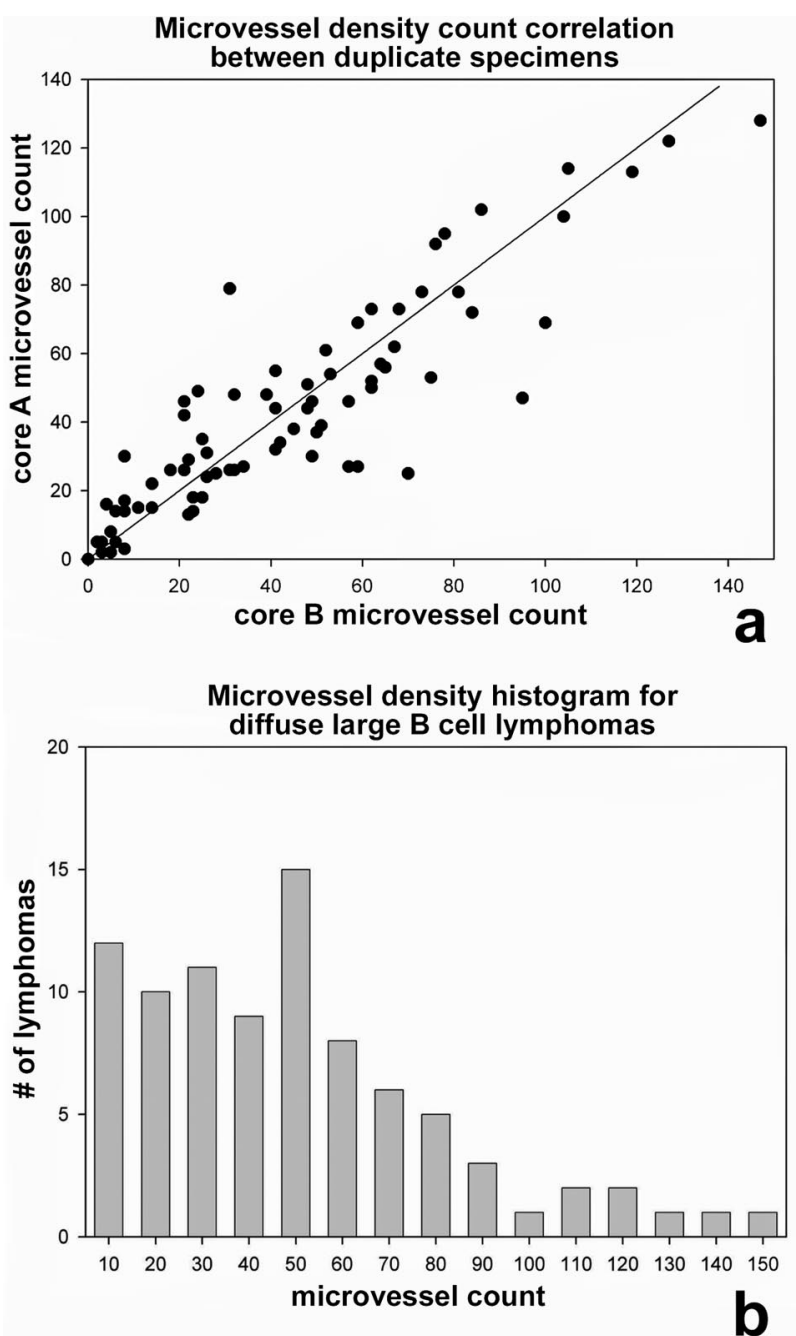

Figure 2. Microvessel density enumeration in DLBCL. a: Histogram of microvessel densities in DLBCL. b: Dot plot of microvessel counts from two separate cores of each lymphoma specimen plotted against each other. The straight line has a slope of 1 , representing perfect correlation.

one another (Figure 2b). A correlation coefficient of 0.87 was obtained, indicating a high degree of correlation between pairs of the same lymphoma cores.

The majority of lymphomas (60\%) showed strong VEGF immunoreactivity, defined as VEGF expression in greater than $30 \%$ of lymphoma cells; the remainder showed either no $(15 \%)$ or weak (25\%) staining (Figure 3, $a$ and b). If VEGF immunohistochemistry is reflective of effective local VEGF signaling, and if local VEGF signaling is an important factor in angiogenesis within DLBCL, we would expect microvessel density to increase with local VEGF expression (Figure 3, c and d). Average microvessel densities did increase with strength of VEGF staining. Average microvessel counts were 20,39 , and 51 vessels per 1.0-mm core for negative, weak, and strong VEGF staining, respectively. These differences were statistically significant $(P<0.007$ for strong versus negative or weak VEGF staining combined and $P<0.05$ for strong versus negative or weak VEGF staining individually; Figure 3e). 

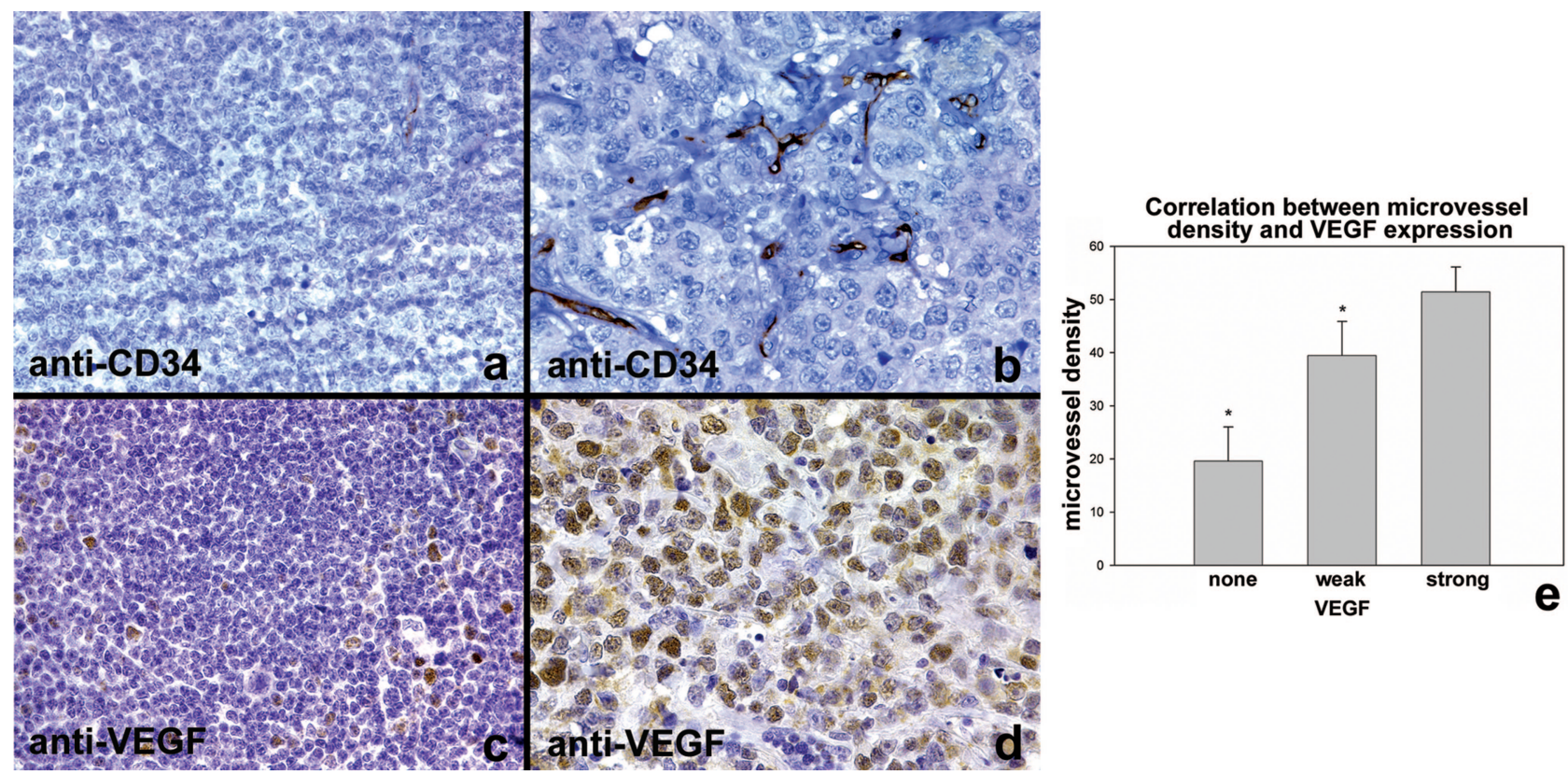

Figure 3. Microvessel density and VEGF expression in DLBCL. Microvessel density [low (a) and high (b)] and VEGF expression [low (c) and high (d)] of corresponding DLBCL cores (anti-CD34, anti-VEGF; magnification, $\times 400$ ). e: Bar graph shows higher microvessel density in DLBCL specimens expressing higher levels of VEGF $\left({ }^{*} P<0.05\right)$

\section{VEGF and Its Receptors VEGFR-1 and VEGFR-2 in DLBCL}

VEGF may play dual roles in the setting of tumorigenesis, through signaling to endothelial cells resulting in angiogenesis (and perhaps vascular permeability) and through signaling to tumor cells as an autocrine/paracrine growth factor. We therefore examined the expression of VEGFR-1 and VEGFR-2 in lymphoma cells (Figure 4, a-d). A $\chi^{2}$ test showed significantly higher expression of both VEGFR-1 and VEGFR-2 in DLBCL specimens with stronger VEGF expression (VEGFR-1, $\chi^{2}=12.6, P<$ 0.002; VEGFR-2, $\chi^{2}=22.8, P=0.00001$ ) (Figure 4, e and f). There was no statistically significant difference in microvascular density in DLBCL specimens with different levels of expression of VEGFR-1 and VEGFR-2 (data not shown).

\section{Immunophenotypic Subgroups of DLBCL}

We have subdivided the DLBCL specimens using nonsupervised hierarchical clustering based on three immunohistochemical markers of GC phenotype (CD10, BCL6, and $\mathrm{HGAL}$ ) and two immunohistochemical markers of non-GC phenotype (MUM1 and BCL2) as previously described. ${ }^{7}$ Clustering reproduced the two expected subgroups, with 40 GC-like cases and 47 non-GC-like cases among the 87 cases for which microvessel density counts were obtainable (Figure 5). We also performed this subclassification using an algorithm devised by Hans et $\mathrm{al}^{12}$ using immunohistochemical staining for CD10, BCL6, and MUM1. This alternate method yielded 42 GClike DLBCL, 41 non-GC-like DLBCL, and four with insufficient data for further categorization. The clustering method and the algorithm by Hans et $\mathrm{al}^{12}$ yielded very similar results, with disparity in only $6 \%$ of cases (data not shown).

DLBCL specimens of both GC-like and non-GC-like subtypes showed a broad range of vascularities, with a weak, nonsignificant trend toward higher values in the GC-like subgroup (average 47 versus 43 vessels per core) (Figure 6a). Expression of both VEGF and its receptors VEGFR-1 and VEGFR-2 showed a broad and similar distribution in both the GC-like and non-GC-like subgroups, with no significant differences (data not shown).

\section{Differences in Vascularity and VEGF and VEGFR Expression in DLBCL Subgroups}

We have shown that in DLBCL, microvessel density is significantly higher in those specimens with higher VEGF expression. We next asked whether this relationship is present in both the GC-like and non-GC-like subgroups. For the purposes of the subgroup analysis, we combined cases that lacked or showed weak staining for VEGF. Interestingly, although in the non-GC-like subgroup, microvessel density remained significantly higher in those specimens with higher VEGF expression (29 versus 53 vessels on average, $P=0.03$ ), the GC-like subgroup showed only a weak nonsignificant trend (40 versus 50 vessels on average, $P=0.27$; Figure $6 \mathrm{~b}$ ). Similar findings were obtained when using the GC-like and non-GC-like subgroups of Hans et al (27 versus 55 vessels on average, $P<0.02$ in the non-GC-like subgroup; 41 versus 49 vessels, $P=0.45$ in the GC-like subgroup; data not shown).

Given the relationship of microvessel density to VEGF expression in non-GC-like but not in GC-like DLBCL, we 

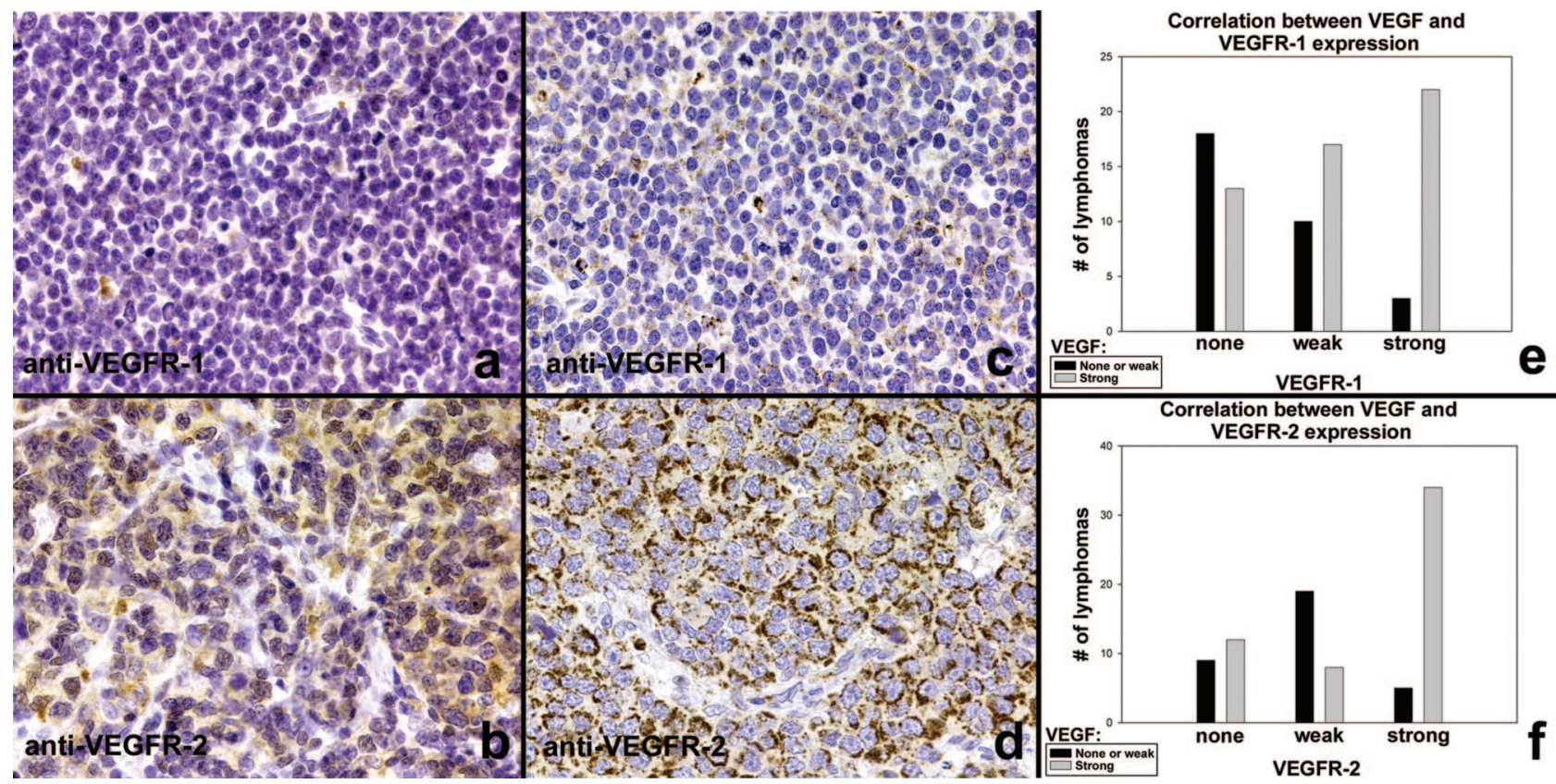

Figure 4. Expression of VEGF and its receptors VEGFR-1 and VEGFR-2 in DLBCL. Low (a and b) and high (c and d) levels of expression of VEGFR-1 (a and c) and VEGFR-2 (b and d) (anti-VEGFR-1, anti-VEGFR-2; $\times 400)$. e: Bar graph showing higher VEGFR-1 expression in DLBCL specimens expressing higher levels of VEGF $(P<0.002)$. f: Bar graph showing higher VEGFR-2 expression in DLBCL specimens expressing higher levels of VEGF $(P=0.00001)$

next examined the relationship between expression of VEGF and its receptors in the two DLBCL subtypes. In both the GC-like and non-GC-like lymphomas, VEGFR-2 expression remained significantly higher in lymphoma specimens expressing higher levels of VEGF (GC-like, $\chi^{2}=15.2, P<0.0005$; non-GC-like, $\chi^{2}=10.2, P=$ 0.006 ) lymphomas (data not shown). Interestingly, however, the same was not true of VEGFR-1 expression in the two DLBCL subtypes. Although in the non-GC-like subgroup of DLBCL, VEGFR-1 expression remained significantly higher in those specimens with higher VEGF expression, the difference was not statistically significant in the GC-like subgroup (non-GC-like, $\chi^{2}=12.6, P<0.002$; GC-like, $\chi^{2}=3.0, P=0.2$ ) (Figure $6, c$ and $d$ ).

\section{Discussion}

Although multiple morphological variants of DLBCL are recognized, no specific histological criteria have been shown to be associated with prognosis, limiting the role of standard histological techniques primarily to those of rendering a diagnosis. In fact, the International Prognostic Index, ${ }^{13}$ which comprises a combination of clinical characteristics, albeit insufficient to capture the heterogeneity of this disease, has been the primary tool used to predict initial response to chemotherapy, freedom from relapse, and overall survival. The roles of angiogenesis and VEGF signaling in hematolymphoid neoplasia are increasingly being recognized. ${ }^{14}$ Using tissue microar-

\section{Diffuse Large B-Cell Lymphoma}
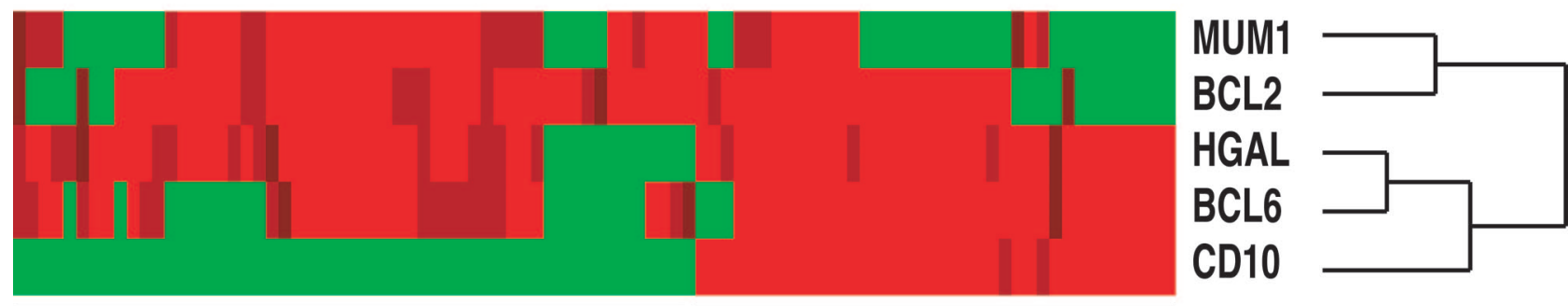

non GC-like

GC-like

Figure 5. Designation of germinal center-like (GC-like) and non-GC-like subgroups of DLBCL using protein expression data. Nonsupervised hierarchical clustering was used to subdivide the DLBCL specimens into a GC-like subgroup preferentially expressing the germinal center-associated proteins CD10, BCL6, and HGAL and a non-GC-like subgroup preferentially expressing the activated B-cell-like proteins MUM1 and BCL2. Strong staining is red, weak staining is lighter red, no staining is green, and uninterpretable staining is black. 

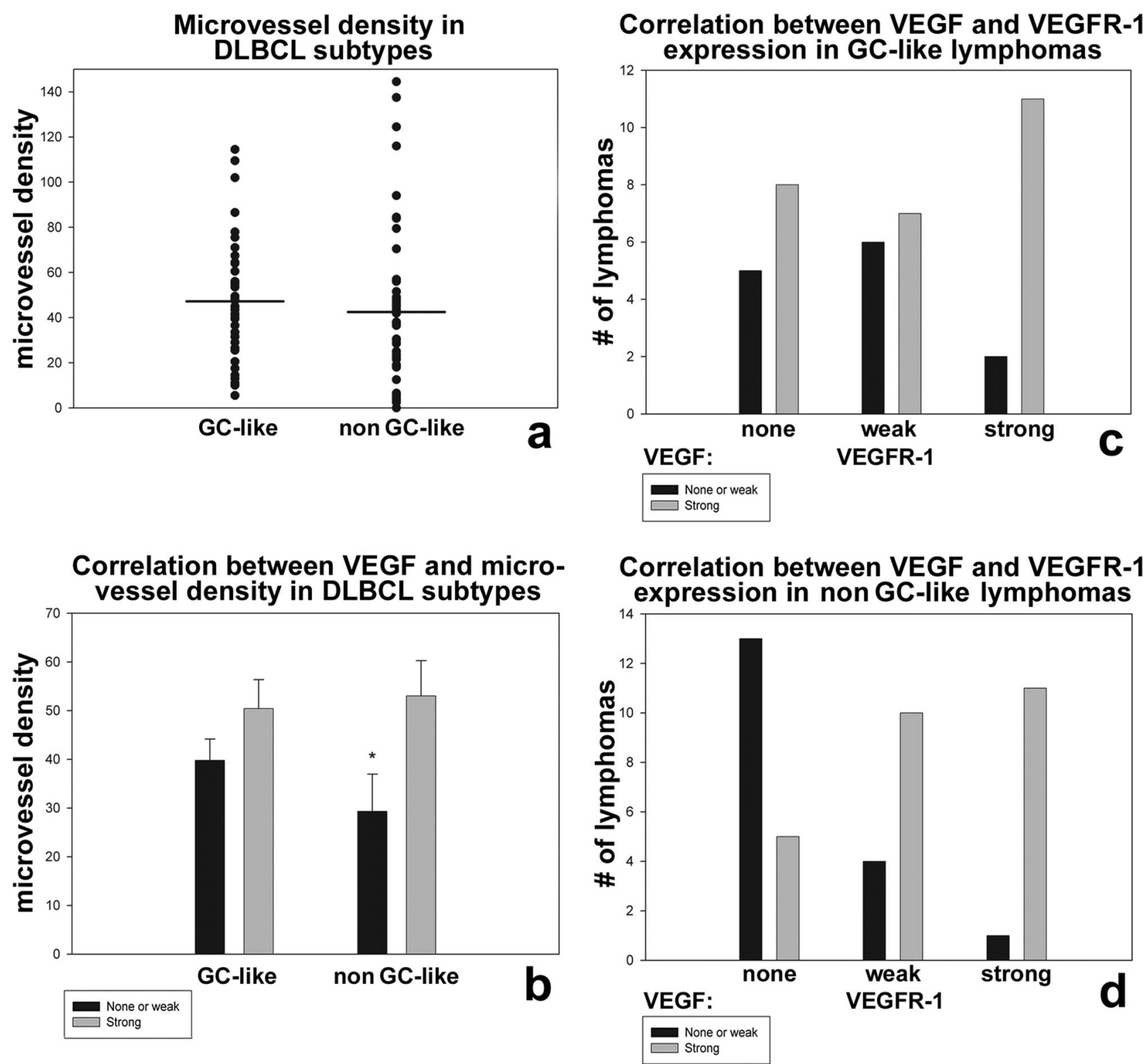

Figure 6. Relationships among vascularity, VEGF expression, and VEGFR-1 expression within GC-like and non-GC-like subgroups of DLBCL. a: Box plot showing no significant difference in microvascular density between subgroups. Each dot represents a single specimen, and the central horizontal bar represents the mean. b: Bar graph showing higher microvessel density in DLBCL specimens expressing higher levels of VEGF in the non-GC-like subgroup but not the GC-like subgroup of DLBCL. ${ }^{*} P=0.03$. $\mathbf{c}$ and $\mathbf{d}$ : Bar graphs showing higher VEGFR-1 expression in DLBCL specimens expressing higher levels of VEGF in the non-GC-like subgroup $(\mathbf{d} ; P<0.002)$ but not the GC-like subgroup $(\mathbf{c})$ of DLBCL.

rays of primary human DLBCL specimens, we used immunohistochemistry to assess vascularity and expression of VEGF and its receptors VEGFR-1 and VEGFR-2 by the lymphoma cells. We have shown that DLBCL has a broad distribution of microvascular densities, which may offer differential access to vascularly distributed nutrients, growth factors, and chemotherapeutics. Furthermore, we have shown that higher microvascular density is present in DLBCL specimens expressing higher levels of VEGF. This finding is consistent with a paracrine role of VEGF elaborated by lymphoma cells in tumor angiogenesis.

VEGF has at least two potential roles in hematolymphoid neoplasia: as an angiogenic/vascular permeability factor mediated through VEGFR-2 and as a survival/proliferation factor mediated through VEGFR-1. In fact, a murine model of human aggressive B-cell non-Hodgkin's lymphoma xenografts demonstrated a role for both autocrine VEGFR-1-mediated signaling in Iymphoma cell proliferation/protection from apoptosis and for paracrine VEGFR-2-mediated angiogenesis. ${ }^{15}$ Interestingly, we have shown that expression of both the VEGFR-1 and VEGFR-2 receptors is higher in DLBCL specimens showing higher expression of their ligand VEGF. These data are compatible with an autocrine role for VEGF in DLBCL, and it is interesting to speculate whether some of the VEGF seen by immunohistochemistry may be present in an active, receptor-bound state. 
GC-like DLBCL

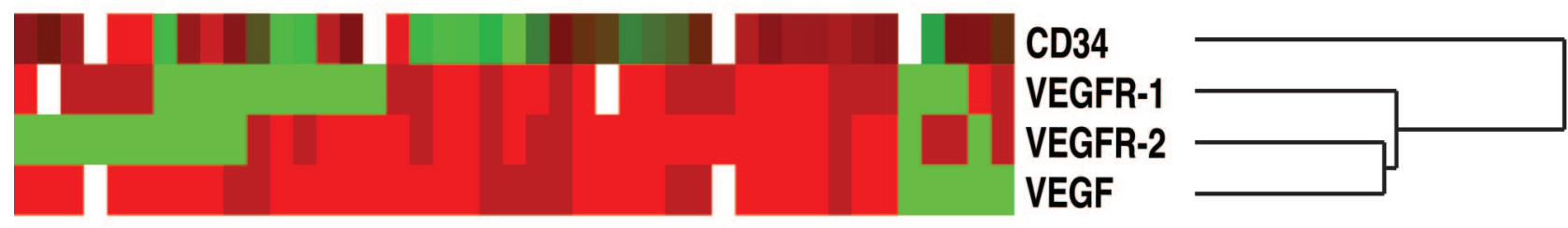

B

\section{Non GC-like DLBCL}
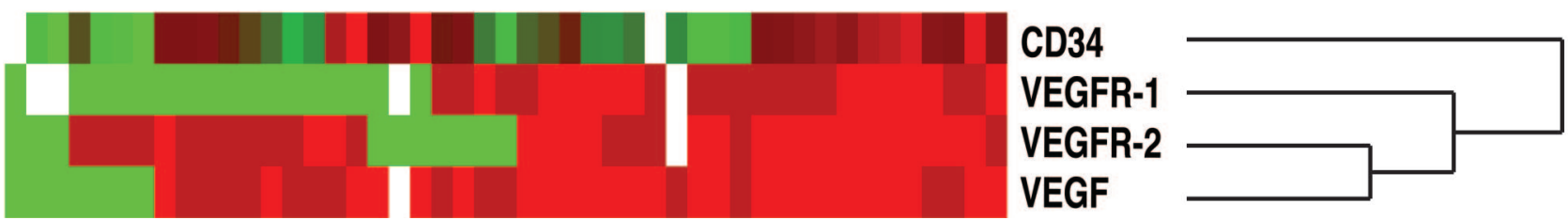

Figure 7. Clustering of GC-like and non-GC-like subgroups of DLBCL according to vascularity markers. Nonsupervised hierarchical clustering was used to visualize covariation of microvascular density and expression of VEGF and its receptors VEGFR-1 and VEGFR-2 in GC-like (A) and non-GC-like (B) DLBCL. For VEGF, VEGFR-1, and VEGFR-2, strong staining is red, weak staining is lighter red, no staining is green, and uninterpretable staining is black. For microvascular density, a continuous scale is used from bright green representing 0 through bright red representing maximum vascularity; gray is present where microvascular densities could not be counted.

Our findings therefore raise the possibility of a dual role for VEGF in lymphomagenesis: as a paracrine angiogenic factor and as an autocrine growth factor. Gene expression profiling of DLBCL has shown VEGF expression to be a poor prognostic factor. ${ }^{16,17}$ VEGF is expressed in isoforms of various lengths depending on splicing ${ }^{2}$; whereas the shortest (121-amino acid) isoform is freely diffusible, the longer isoforms bind matrix components and thus are more likely to be locally retained. Thus, although it seems likely that VEGF mRNA levels correlate with locally retained VEGF protein as assessed by immunohistochemistry, outcome studies are needed to assess whether the poor prognosis associated with increasing VEGF mRNA levels is recapitulated by VEGF protein expression levels as measured by immunohistological evaluation.

We further evaluated the relationships among vascularity and VEGF/VEGFR expression within the GC-like and non-GC-like subgroups of lymphomas in our dataset, using hierarchical clustering of a five-component immunoprofile. ${ }^{7}$ The same heterogeneity of vascularity seen in DLBCL is present within the GC-like and the non-GC-like subgroups of DLBCL. One might expect that increased microvessel density would correlate with aggressive clinical behavior; however, we have found no significant difference in average microvessel densities between the two subgroups. The role of microvessel density may vary according to the type of hematolymphoid neoplasm, because it represents the route of delivery not only of nutrients and growth factors but also of chemotherapeutic agents. In the extensively studied setting of multiple myeloma, microvessel density correlates both with progression of the disease ${ }^{18}$ and poorer overall survival. ${ }^{19}$ By contrast, however, a prospective study of follicular lymphomas has shown increased microvessel density to be an independent favorable prognostic factor for overall survival. ${ }^{20}$

Serum levels of VEGF have not been found to be different between GC-like and non-GC-like DLBCL subtypes. ${ }^{21}$ We have similarly found no significant difference in VEGF expression between the two lymphoma subtypes. However, we found that only in the non-GC-like subgroup of DLBCL is the microvascular density higher in specimens that express higher levels of VEGF. One might speculate therefore that the paracrine role of VEGF in tumor angiogenesis is more significant in non-GC-like than in GC-like lymphomas. Furthermore, VEGFR-1 expression is higher in specimens showing higher levels of VEGF expression in the non-GC-like subtype of DLBCL but not in the GC-like subtype. This difference is substantiated by nonsupervised hierarchical clustering (Figure 7), which demonstrates close clustering of vascularity markers in the non-GC-like subtype in comparison with the GC-like subtype. Clearly, future work is warranted to assess whether DLBCL with higher microvascular density and/or higher expression of VEGF or its receptors represents a prognostically or therapeutically distinct subgroup.

\section{References}

1. Podar K, Anderson KC: The pathophysiologic role of VEGF in hematologic malignancies: therapeutic implications. Blood 2005, 105:1383-1395

2. Cross MJ, Dixelius J, Matsumoto T, Claesson-Welsh L: VEGF-receptor signal transduction. Trends Biochem Sci 2003, 28:488-494

3. Gerber HP, Malik AK, Solar GP, Sherman D, Liang XH, Meng G, Hong K, Marsters JC, Ferrara N: VEGF regulates haematopoietic stem cell survival by an internal autocrine loop mechanism. Nature 2002, 417:954-958 
4. Bellamy WT, Richter L, Frutiger Y, Grogan TM: Expression of vascular endothelial growth factor and its receptors in hematopoietic malignancies. Cancer Res 1999, 59:728-733

5. Armitage JO, Weisenburger DD: New approach to classifying nonHodgkin's lymphomas: clinical features of the major histologic subtypes. Non-Hodgkin's Lymphoma Classification Project. J Clin Oncol 1998, 16:2780-2795

6. Alizadeh AA, Eisen MB, Davis RE, Ma C, Lossos IS, Rosenwald A, Boldrick JC, Sabet H, Tran T, Yu X, Powell JI, Yang L, Marti GE, Moore T, Hudson Jr J, Lu L, Lewis DB, Tibshirani R, Sherlock G, Chan WC, Greiner TC, Weisenburger DD, Armitage JO, Warnke R, Levy R, Wilson W, Grever MR, Byrd JC, Botstein D, Brown PO, Staudt LM: Distinct types of diffuse large B-cell lymphoma identified by gene expression profiling. Nature 2000, 403:503-511

7. Natkunam Y, Lossos IS, Taidi B, Zhao S, Lu X, Ding F, Hammer AS, Marafioti T, Byrne Jr GE, Levy S, Warnke RA, Levy R: Expression of the human germinal center-associated lymphoma (HGAL) protein, a new marker of germinal center B-cell derivation. Blood 2005, 105:3979-3986

8. Kononen J, Bubendorf L, Kallioniemi A, Barlund M, Schraml P, Leighton S, Torhorst J, Mihatsch MJ, Sauter G, Kallioniemi OP: Tissue microarrays for high-throughput molecular profiling of tumor specimens. Nat Med 1998, 4:844-847

9. Weidner N, Semple JP, Welch WR, Folkman J: Tumor angiogenesis and metastasis: correlation in invasive breast carcinoma. $N$ Engl J Med 1991, 324:1-8

10. Page RD: TreeView: an application to display phylogenetic trees on personal computers. Comput Appl Biosci 1996, 12:357-358

11. Eisen MB, Spellman PT, Brown PO, Botstein D: Cluster analysis and display of genome-wide expression patterns. Proc Natl Acad Sci USA: 1998, 95:14863-14868

12. Hans CP, Weisenburger DD, Greiner TC, Gascoyne RD, Delabie J, Ott G, Muller-Hermelink HK, Campo E, Braziel RM, Jaffe ES, Pan Z, Farinha P, Smith LM, Falini B, Banham AH, Rosenwald A, Staudt LM, Connors JM, Armitage JO, Chan WC: Confirmation of the molecular classification of diffuse large B-cell lymphoma by immunohistochemistry using a tissue microarray. Blood 2004, 103:275-282

13. Shipp MA, Harrington DP, Anderson JR, Armitage JO, Bonadonna G, Brittinger G, Cabanillas F, Canellos GP, Coiffier B, Connors JM,
Cowan RA, Crowther D, Dahlberg S, Engelhard M, Fisher RI, Gisselbrecht C, Horning SJ, Lepage E, Lister TA, Meerwaldt JH, Montserrat E, Nissen NI, Oken MM, Peterson BA, Tondini C, Velasquez WA, Yeap BY: A predictive model for aggressive non-Hodgkin's lymphoma: The International Non-Hodgkin's Lymphoma Prognostic Factors Project. N Engl J Med 1993, 329:987-994

14. Koster A, Raemaekers JM: Angiogenesis in malignant lymphoma. Curr Opin Oncol 2005, 17:611-616

15. Wang ES, Teruya-Feldstein J, Wu Y, Zhu Z, Hicklin DJ, Moore MA: Targeting autocrine and paracrine VEGF receptor pathways inhibits human lymphoma xenografts in vivo. Blood 2004, 104:2893-2902

16. Shipp MA, Ross KN, Tamayo P, Weng AP, Kutok JL, Aguiar RC, Gaasenbeek M, Angelo M, Reich M, Pinkus GS, Ray TS, Koval MA Last KW, Norton A, Lister TA, Mesirov J, Neuberg DS, Lander ES, Aster JC, Golub TR: Diffuse large B-cell lymphoma outcome prediction by gene-expression profiling and supervised machine learning. Nat Med 2002, 8:68-74

17. Salven $P$, Teerenhovi L, Joensuu H: A high pretreatment serum vascular endothelial growth factor concentration is associated with poor outcome in non-Hodgkin's lymphoma. Blood 1997, 90:3167-3172

18. Vacca A, Ribatti D, Roncali L, Ranieri G, Serio G, Silvestris F, Dammacco $\mathrm{F}$ : Bone marrow angiogenesis and progression in multiple myeloma. Br J Haematol 1994, 87:503-508

19. Kumar S, Gertz MA, Dispenzieri A, Lacy MQ, Wellik LA, Fonseca R, Lust JA, Witzig TE, Kyle RA, Greipp PR, Rajkumar SV: Prognostic value of bone marrow angiogenesis in patients with multiple myeloma undergoing high-dose therapy. Bone Marrow Transplant 2004, 34:235-239

20. Koster A, van Krieken JH, Mackenzie MA, Schraders M, Borm GF van der Laak JA, Leenders W, Hebeda K, Raemaekers JM: Increased vascularization predicts favorable outcome in follicular lymphoma. Clin Cancer Res 2005, 11:154-161

21. Pedersen LM, Jurgensen GW, Johnsen HE: Serum levels of inflammatory cytokines at diagnosis correlate to the bcl-6 and CD10 defined germinal centre (GC) phenotype and bcl-2 expression in patients with diffuse large B-cell lymphoma. $\mathrm{Br} J$ Haematol 2005, 128:813-819 